\title{
Positive Radial Solutions for Semilinear Biharmonic Equations in Annular Domains
}

\author{
ROBERT DALMASSO
}

ABSTRACT. We study the existence of positive radial solutions of $\Delta^{2} u=g(|x|) f(u)$ in an annulus with Dirichlet boundary conditions. We establish that the equation has at least one positive radially symmetric solution on any annulus if $f$ and $g$ are nonnegative, $g \neq 0$ and $f$ is superlinear at zero and $+\infty$. We also give a property of positive radial solutions.

\section{INTRODUCTION}

In this paper we consider the existence of positive radial solutions of the semilinear biharmonic equation

$$
\begin{array}{ll}
\Delta^{2} u=g(|x|) f(u) & \text { in } \Omega(a, b) \\
u=\frac{\partial u}{\partial v}=0 & \text { on } \partial \Omega(a, b)
\end{array}
$$

where $0<a<b<+\infty, \Omega(a, b)$ denotes the annulus $\left\{x \in \mathbb{R}^{n} ; a<|x|<b\right\}$ $(n \geq 2), \frac{\partial}{\partial \nu}$ is the outward normal derivative and $f, g$ satisfy the following hypotheses

$\left(H_{1}\right) \quad f \in C([0,+\infty))$ and $f(u) \geq 0$ for $u>0$

$\left(H_{2}\right) \quad g \in C([a, b]), g(r) \geq 0$ for $r \in[a, b]$ and $g \equiv 0$ in $[a, b]$.

1991 Mathematics Subject Classification: 35B45, 35J40, $35 \mathrm{~J} 65$.

Editorial Complutense. Madrid, 1993. 


$$
\begin{aligned}
& \left(H_{3}\right) \quad \lim _{u \rightarrow+\infty} f(u) / u=+\infty . \\
& \left(H_{4}\right) \quad \lim _{u \rightarrow 0} f(u) / u=0 .
\end{aligned}
$$

The analogous problem for the Laplace equation has been intensively studied in recent years (see e.g. [1]-[4], [8], [11], [12], [15]) and nearly optimal results have been obtained. In most papers, the shooting method was used to establish the existence of positive radial solutions. In contrast the result of [1] was obtained by a variational method and the use of a priori estimates, while in [15] an expansion fixed point theorem was applied.

Our main result is the following theorem.

Theorem 1.1. Assume $\left(H_{1}\right)-\left(H_{4}\right)$. Then problem $(1.1)$, (1.2) possesses at least one positive radial solution $\left.u \in C^{4}(\overline{\Omega(a, b})\right)$.

In this paper our method of proof makes use of a priori estimates and well-known properties of compact mappings taking a cone in a Banach space into itself (see [7]).

Since we are interested in positive radial solutions, the problem under consideration reduces to the one-dimensional boundary value problem

$$
\begin{array}{ll}
\Delta^{2} u(t)=g(t) f(u(t)), & t \in(a, b) \\
u^{(j)}(a)=u^{(j)}(b)=0, & j=0,1
\end{array}
$$

where $\Delta$ denotes the polar form of the Laplacian, i.e. $\Delta=$ $\mathrm{t}^{1-n} \frac{d}{d t}\left(t^{n-1} \frac{d}{d t}\right)$.

Our next result gives a property of nonnegative nontrivial solutions of (1.3), (1.4) when $f$ and $g$ satisfy some monotonicity conditions.

Theorem 1.2. Suppose that $f$ and $g$ in equation (1.3) satisfy the following assumptions:

( $\left.H_{5}\right) \quad f:[0,+\infty) \rightarrow[0,+\infty)$ is nondecreasing.

$\left(H_{6}\right) \quad g:[a, b] \rightarrow[0,+\infty)$ is nonincreasing. 
Let $u \in C^{4}([a, b])$ be a nonnegative nontrivial solution of problem (1.3), (1.4). Then $\Delta u(a)>\Delta u(b)$.

Remark 1.1. Note that $\Delta u(a)=u^{\prime \prime}(a)$ (resp. $\Delta u(b)=\mathrm{u}^{\prime \prime}(b)$ ) since $u^{\prime}(a)=0$ (resp. $\left.u^{\prime}(b)=0\right)$.

Remark 1.2. Theorems 1.1 and 1.2 can be easily extended to handle more general nonlinearities of the type $f(|x|, u)$.

Our paper is organized as follows. In Section 2 we give a maximum principle for fourth order equations and we describe the special shape of nontrivial solutions of (1.3), (1.4) when $f \geq 0$ and $g \geq 0$. In Section 3 we prove our a priori bounds for positive solutions of (1.3), (1.4). Theorem 1.1 is proved in Section 4. Finally, Section 5 contains the proof of theorem 1.2.

\section{PRELIMINARIES}

We have the following theorem.

Theorem 2.1. Let $u \in C^{4}([a, b])$ be such that

$$
\left\{\begin{array}{l}
\Delta^{2} u \geq 0 \text { in }(a, b) \\
u^{(j)}(a)=u^{(j)}(b)=0, j=0,1 .
\end{array}\right.
$$

Assume that $u \neq 0$. Then:

(i) There exist $r, s \in(a, b)$ such that $r<s, \Delta u>0$ on $[a, r) \cup(s, b]$ and $\Delta u<0$ on $(r, s)$.

(ii) There exist $d_{1}, d_{2} \in(r, s)$ such that $d_{1} \leq d_{2},(\Delta u)^{\prime}<0$ on $\left[a, d_{1}\right)$, $(\Delta u)^{\prime}>0$ on $\left(d_{2}\right.$, b] and $(\Delta u)^{\prime} \equiv 0$ on $\left[d_{1}, d_{2}\right]$.

(iii) $u>0$ on $(a, b)$. Moreover there exists $c \in(r, s)$ such that $u^{\prime}>0$ on $(a, c)$ and $u^{\prime}<0$ on $(c, b)$.

Proof. We first prove (i). Suppose that $\Delta u(a) \leq 0$ and $\Delta u(b) \leq 0$. Then the one-dimensional maximum principle ([14] p. 2) implies that $\Delta u \leq 0$ on $[a, b]$. Since $u(a)=u^{\prime}(a)=u(b)=u^{\prime}(b)=0$, the maximum prin- 
ciple and the Hopf boundary lemma ([14] p. 4) imply that $u \equiv 0$ on $[a, b]$ and we reach a contradiction. Thus $\Delta u(a)>0$ or $\Delta u(b)>0$. Suppose for instance that $\Delta u(a)>0$. If $\Delta u \geq 0$ on $[a, b]$, we get a contradiction as before. Thus there exists $x \in(a, b)$ such that $\Delta u(x)<0$ and we can define $r \in(a, b)$ to be the first zero of $\Delta u$ in $(a, b)$. Since $u^{\prime \prime}(a)=\Delta u(a)>0$ we have $u>0$ in $(a, a+\eta]$ for some $\eta>0$. Using the maximum principle and the Hopf lemma we get $u^{\prime}>0$ on $(a, r]$. Now, if $\Delta u(b) \leq 0$, the maximum principle implies $\Delta u<0$ on $(r, b)$. Since $u(r)>0$ and $u(b)=u^{\prime}(b)=0$, we again reach a contradiction. Thus we have proved that $\Delta u(b)>0$. Now we can define $s \in(r, b)$ to be the last zero of $\Delta u$ in $(a, b)$. Since $\Delta u(x)<0$, the maximum principle implies that $\Delta u<0$ on $(r, s)$.

We now prove (ii). Denoting by $m<0$ the minimum value of $\Delta u$ in $[a, b]$, we define $E=\{t \in(a, b) / \Delta u(t)=m\}$. Suppose first that $E$ contains only one point. Then with the aid of the Hopf lemma we obtain (ii). Now, if $E$ contains at least two points, the maximum principle and the continuity of $\Delta u$ imply that $E=\left[d_{1}, d_{2}\right]$ where $r<d_{1}<d_{2}<s$. Then, using the Hopf lemma we obtain (ii).

Finally we prove (iii). We have already seen that $u^{\prime}>0$ on $(a, r]$. In the same way we show that $u^{\prime}<0$ on $[s, b)$. Now let $t_{0}$ (resp. $t_{1}$ ) be the first (resp. the last) zero of $u^{\prime}$ in $(a, b)$. Clearly $r<t_{0} \leq t_{1}<s$. Suppose that $t_{0}<t_{1}$. Then the Hopf lemma implies that either $u^{\prime}\left(t_{0}\right)>0$ or $u^{\prime}\left(t_{1}\right)<0$, a contradiction. Thus $t_{0}=t_{1}$ and (iii) is proved.

\section{A PRIORI BOUNDS}

Theorem 3.1. Assume $\left(H_{1}\right)-\left(H_{3}\right)$. Then there exists $M>0$ such that

$$
\|u\|_{\infty} \leq M
$$

for all positive solutions $u \in C^{4}([a, b])$ of $(1.3)$, (1.4).

Proof. We denote by $S$ the set of all positive solutions of (1.3), (1.4) in $C^{4}([a, b])$. Let $u \in S$. By theorem 2.1 there exist $c(u), d_{1}(u), d_{2}(u)$, $r(u)$ and $s(u)$ in $(a, b)$ such that $u^{\prime}>0$ on $(a, c(u)), u^{\prime}<0$ on $(c(u), b)$, $(\Delta u)^{\prime}<0$ on $\left[a, d_{1}(u)\right),(\Delta u)^{\prime}>0$ on $\left(d_{2}(u), b\right],(\Delta u)^{\prime} \equiv 0$ on $\left[d_{1}, d_{2}\right]$, $\Delta u>0$ on $[a, r(u)) \cup(s(u), b]$ and $\Delta u<0$ on $(r(u), s(u))$. Moreover we have $a<r(u)<c(u), d_{1}(u), d_{2}(u)<s(u)<b$ and $d_{1}(u) \leq d_{2}(u)$.

We shall divide the proof into several steps. Subsequently $C$ will denote various generic constants which may vary from line to line. 
Step 1. We first prove that $\{g f(u) ; u \in S\}$ and $\{g u ; u \in S\}$ are bounded in $L_{\mathrm{loc}}^{1}(a, b)$. Define

$$
\varrho(t)=(t-a)^{2}(t-b)^{2} \quad \text { for } a \leq t \leq b .
$$

Let $\varphi \in C^{4}([a, b])$ be the solution of the boundary problem

$$
\left\{\begin{array}{l}
\Delta^{2} \varphi=g Q \quad \text { in }(a, b) \\
\varphi^{(j)}(a)=\varphi^{(j)}(b)=0, \quad j=0,1 .
\end{array}\right.
$$

By theorem $2.1 \varphi>0$ in $(a, b)$ and there exist $c_{1}>0$ and $c_{2}>0$ such that

$$
c_{1} \varrho \leq \varphi \leq c_{2} \varrho \quad \text { on }[a, b] \text {. }
$$

By $\left(H_{3}\right)$, there exists $\lambda>c_{1}^{-1}$ and $u_{0} \geq 0$ such that

$$
f(u) \geq \lambda u \quad \text { for } u \geq u_{0} .
$$

If we multiply equation (1.3) by $t^{n-1} \varphi$ and integrate by parts four times we obtain

$$
\int_{a}^{b} t^{n-1} \varphi g f(u) d t=\int_{a}^{b} t^{n-1} \varrho g u d t .
$$

From (3.2) and (3.3) we deduce

$$
\int_{a}^{b} t^{n-1} \varrho g u d t \geq \lambda \int_{a}^{b} t^{n-1} \varphi g u d t-C \geq \lambda c_{1} \int_{a}^{b} t^{n-1} \varrho g u d t-C
$$

that is

$$
\begin{gathered}
\int_{a}^{b} t^{n-1} \varrho g u d t \leq \frac{C}{\lambda c_{1}-1} \\
\int_{a}^{b} t^{n-1} \varrho g f(u) d t \leq \frac{C}{c_{1}\left(\lambda c_{1}-1\right)} .
\end{gathered}
$$

Thus $\{g f(u) ; u \in S\}$ and $\{g u ; u \in S\}$ are bounded in $L_{l o c}^{1}(a, b)$.

Step 2. Now we prove the following lemma. 
Lemma 3.1. Let $A$ be a subset of $S$. Then:

(i) If $\{\Delta u ; u \in A\}$ is bounded in $L^{1}(a, b)$, then there exists a constant $M>0$ such that $\|u\|_{\infty} \leq M$ for all $u \in A$.

(ii) If there exist $\gamma>0, \eta>0$ and $C>0$ such that $\gamma+\eta \leq b-a$ and $u(t) \leq C$ for $t \in[a, a+\gamma] \cup[b-\eta, b]$ and $u \in A$, then there exists a constant $M \geq C$ such that $\|u\|_{\infty} \leq M$ for all $u \in A$.

Proof. (i) follows readily from the fact that $u$ and $u^{\prime}$ vanish at least once in $[a, b]$. We now prove (ii). Setting $m=\inf _{[a+\gamma, b-\eta]} \varrho(t)$ and using (3.5) we obtain

$$
\begin{aligned}
\int_{a}^{b} t^{n-1} \Delta^{2} u d t & =\int_{a}^{b} t^{n-1} g f(u) d t=\int_{a}^{++y}+\int_{a+\gamma}^{b-\eta}+\int_{b-\eta}^{b} \\
& \leq C+\int_{a+\gamma}^{b-\eta} t^{n-1} \frac{1}{m} \varrho g f(u) d t \\
& \leq C\left(1+\int_{a}^{b} t^{n-1} \varrho g f(u) d t\right) \leq C .
\end{aligned}
$$

Thus $\left\{\Delta^{2} u ; u \in A\right\}$ is bounded in $L^{1}(a, b)$. Since $u, u^{\prime}, \Delta u$ and $(\Delta u)^{\prime}$ vanish at least once in $[a, b]$ the result follows.

Step 3. Finally we prove that $S$ is bounded in $L^{\infty}(a, b)$. Let $\gamma>0$, $\eta>0$ and $\delta>0$ be such that $\gamma+\eta+2 \delta<b-a$ and $g>0$ on $[a+\gamma, b-\eta]$. Define $a^{\prime}=a+\gamma+\delta$ and $b^{\prime}=b-\eta-\delta$. Then by (3.4) there exists $K>0$ such that

$$
\int_{a^{\prime}}^{b^{\prime}} u d t \leq K .
$$

Let $n_{0}>0$ be such that $1 / n_{0}<\left(b^{\prime}-a^{\prime}\right) / 4$. By (3.6) we have

$$
\text { meas }\left\{t \in\left[a^{\prime}, b^{\prime}\right] ; u(t) \geqslant n_{0} K\right\} \leq \frac{1}{n_{0}}<\frac{b^{\prime}-a^{\prime}}{4} \text {. }
$$


Now define

$$
\begin{aligned}
& S_{+}=\left\{u \in S ; c(u)>b^{\prime}\right\} \\
& S_{-}=\left\{u \in S ; c(u)<a^{\prime}\right\}
\end{aligned}
$$

and

$$
\dot{S_{0}}=\left\{u \in S ; a^{\prime} \leq c(u) \leq b^{\prime}\right\} .
$$

Thus $S=S_{-} \cup S_{0} \cup S_{+}$. Let $\alpha=a^{\prime}+\left(b^{\prime}-a^{\prime}\right) / 4$ and $\beta=b^{\prime}-\left(b^{\prime}-a^{\prime}\right) / 4$. Clearly by the shape of $u,(3.7)$ implies

$$
u(t)<n_{0} K \text { for } t \in[a, \beta] \text { and } u \in S_{+}
$$

and

$$
u(t)<n_{0} K \text { for } t \in[\alpha, b] \text { and } u \in S_{-} .
$$

Lemma 3.2. $S_{0}$ is bounded in $L^{\infty}(a, b)$.

Proof. Let $u \in S_{0}$. From (3.4) we get

$$
C \geq \int_{a+\gamma}^{b-\eta} u d t \geq \int_{a+\gamma}^{a^{\prime}} u d t+\int_{b^{\prime}}^{b-\eta} u d t
$$

which implies

$$
u(t) \leq C / \delta \text { for } t \in[a, a+\gamma] \cup[b-\eta, b]
$$

and we get the conclusion by using lemma 3.1 (ii).

Clearly Theorem 3.1 follows from Lemma 3.2 and the next lemma.

Lemma 3.3. $\underline{S}_{+}$and $\underline{S}$ are bounded in $L^{\infty}(a, b)$.

Proof. We shall show that $S_{-}$is bounded in $L^{\infty}(a, b)$. We first prove that $F=\left\{u \in S_{-} ; s(u)>\beta\right\}$ is bounded in $L^{\infty}(a, b)$. We claim that for $u \in F$ 


$$
\|u\|_{\infty} \leq M=n_{0} K+T(b-a)
$$

where $T=(2 /(\beta-\alpha)) n_{0} K(b / a)^{n-1}$. Indeed, suppose this is not the case. Then let $u \in F$ be such that $u(c(u))>M$. We have $c(u)<\alpha$. Let $t \in[\alpha, \beta]$ be arbitrary. By the mean value theorem there exists $x \in(c(u), t)$ such that, by virtue of $(3.9)$

$$
u^{\prime}(x)=\frac{u(t)-u(c(u))}{t-c(u)}<-T .
$$

Since $r(u)<c(u)<\alpha<\beta<s(u), y^{n-1} u^{\prime}(y)$ is nonincreasing on $[x, t]$, thus we get

$$
u^{\prime}(t) \leq\left(\frac{x}{t}\right)^{n-1} u^{\prime}(x)<-\left(\frac{a}{b}\right)^{n-1} T
$$

with $t \in[\alpha, \beta]$. This and (3.9) imply

$$
u(t)<n_{0} K-T\left(\frac{a}{b}\right)^{n-1}(t-\alpha) \quad \text { for } t \in[\alpha, \beta] .
$$

For $t \in[(\alpha+\beta) / 2, \beta]$, we deduce

$$
u(t)<n_{0} K-T\left(\frac{a}{b}\right)^{n-1} \frac{\beta-\alpha}{2}=0
$$

and we reach a contradiction. Thus our assertion is proved

Now we prove that $G=\left\{u \in S_{-} ; s(u) \leq \beta\right\}$ is bounded in $L^{\infty}(a, b)$. Let $t \in[\beta, b)$. We first show that $H=\{\Delta u(t) ; u \in G\}$ is bounded. Suppose $n \geq 3$. Since $\Delta u(s) \geq \Delta u(t) \geq 0$ for $s \in[t, b]$, we have

$$
u(t)=\frac{1}{n-2} \int_{t}^{b}\left(\left(\frac{s}{t}\right)^{n-2}-1\right) s \Delta u(s) d s \geq C(t) \Delta u(t)
$$

where $C(t)>0$ does not depend on $u$. Thus, if $H$ is not bounded we get a contradiction with (3.9). When $n=2$ the argument is the same.

Now let $\chi \in C^{4}([\beta, b])$ be the solution of the boundary problem

$$
\left\{\begin{array}{l}
\Delta^{2} \chi=1 \text { in }(\beta, b) \\
\chi^{(j)}(\beta)=\chi^{(j)}(b)=0, j=0,1 .
\end{array}\right.
$$


Multiplying equation (1.3) by $t^{n-1} \chi$ and integrating by parts four times we get

$$
\begin{aligned}
\int_{t}^{b} s^{n-1} \chi g f(u) d s & =\int_{t}^{b} s^{n-1} \chi \Delta^{2} u d s=-t^{n-1} \chi(t)(\Delta u)^{\prime}(t) \\
& +t^{n-1} \chi^{\prime}(t) \Delta u(t)-t^{n-1} \Delta \chi(t) u^{\prime}(t) \\
& +t^{n-1}(\Delta \chi)^{\prime}(t) u(t)+\int_{t}^{b} s^{n-1} u d s
\end{aligned}
$$

for all $t \in[\beta, b)$. Setting $t=\beta$ in (3.10) we obtain

$$
\int_{\beta}^{b} s^{n-1} \chi g f(u) d s=-\beta^{n-1} \Delta \chi(\beta) u^{\prime}(\beta)+\beta^{n-1}(\Delta \chi)^{\prime}(\beta) u(\beta)+\int_{\beta}^{b} s^{n-1} u d s .
$$

Since by Theorem $2.1 \Delta \chi(\beta)>0$, we deduce from (3.9) and (3.11) that $\left\{u^{\prime}(\beta) ; u \in G\right\}$ is bounded. Since $\Delta u>0$ on $(\beta, b]$ when $u \in G$, we have $\beta^{n-1} u^{\prime}(\beta)<t^{n-1} u^{\prime}(t)<0$ for $t \in(\beta, b)$. Hence $\left\{u^{\prime}(s) ; u \in G, s \in[\beta, b]\right\}$ is bounded. From this, (3.9), (3.10), Theorem 2.1 and the fact that $H=\{\Delta u(t) ; u \in G\}$ is bounded for each fixed $t \in[\beta, b)$ we deduce that $\left\{(\Delta u)^{\prime}(t) ; u \in G\right\}$ is bounded for each fixed $t \in(\beta, b)$. Since for $t \in(\beta, b)$ we have

$$
b^{n-1}(\Delta u)^{\prime}(b)-t^{n-1}(\Delta u)^{\prime}(t)=\int_{t}^{b} s^{n-1} g f(u) d s
$$

we obtain that $\left\{(\Delta u)^{\prime}(b) ; u \in G\right\}$ is bounded. Now let $d$ be such that $d_{1}(u) \leq d \leq d_{2}(u)$. Using the fact that $t^{n-1}(\Delta u)^{\prime}$ is nondecreasing on $[a, b]$ we can write

$$
0=d^{n-1}(\Delta u)^{\prime}(d) \leq t^{n-1}(\Delta u)^{\prime}(t) \leq b^{n-1}(\Delta u)^{\prime}(b)
$$

for $t \in[d, b]$, from which we deduce that $\left\{(\Delta u)^{\prime}(t) ; u \in G, t \in[d, b]\right\}$ is bounded. Since

$$
\Delta u(b)=\int_{s(u)}^{b}(\Delta u)^{\prime} d s
$$


we obtain that $\{\Delta u(b) ; u \in G\}$ is bounded. Now we write

$$
\Delta u(d)=\Delta u(b)-\int_{d}^{b}(\Delta u)^{\prime} d s
$$

and we finally obtain that $\{\Delta u(t) ; u \in G, t \in[d, b]\}$ is bounded. Using the fact that

$$
\int_{a}^{b} t^{n-1} \Delta u d t=0
$$

for all $u \in S$, we easily deduce that $\{\Delta u ; u \in G\}$ is bounded in $L^{1}(a, b)$ and the conclusion follows from lemma 3.1 (i).

It remains to prove that $S_{+}$is bounded in $L^{\infty}(a, b)$. The proof is similar. Using analogous arguments we show that $\left\{u \in S_{+} ; r(u)<\alpha\right]$ and $\left\{u \in S_{+} ; r(u) \geq \alpha\right\}$ are bounded in $L^{\infty}(a, b)$. The proof of lemma 3.3 is complete.

Remark 3.1. Note that the constant $M$ in theorem 3.1 can be chosen independently of the parameter $x \in\left[0, x_{0}\right]$ for each fixed $x_{0} \in(0,+\infty)$ if we consider positive solutions of (1.3), (1.4) for the family of nonlinearities $f_{x}(t)=f(t+x), t \geq 0$.

\section{PROOF OF THEOREM 1.1}

We shall prove that problem (1.3), (1.4) has at least one positive solution $u \in C^{4}([a, b])$. The proof makes use of the Kranosel'skii type fixed point theorem [7] (proposition 2.1 and remark 2.1).

The homogeneous Dirichlet problem

$$
\left\{\begin{array}{l}
\Delta^{2} v=0 \text { in }(a, b) \\
v^{(j)}(a)=v^{(j)}(b)=0, j=0,1
\end{array}\right.
$$

has only the trivial solution. Then it is well-known (see e.g. [13] p. 29) that the operator $\Delta^{2}$ with Dirichlet boundary conditions has one and only one Green's function $G(t, s)$. Define the closed cone 


$$
Z=\{u \in C([a, b]) ; u \geq 0\} .
$$

For $(u, x) \in Z \times[0,+\infty)$ we define

$$
F(u, x)(t)=\int_{a}^{b} G(t, s) g(s) f(u(s)+x) d s
$$

and

$$
\Phi(u)=F(u, 0) .
$$

By Theorem $2.1 F$ maps $Z \times[0,+\infty)$ into $Z$. Since $G$ is continuous, it is well-known that $F$ is compact. Now the following properties hold:

(i) By Theorem 2.1 and the properties of the Green's function any nontrivial solution of the fixed point equation

$$
\Phi(u)=u, \quad u \in Z,
$$

yields a positive solution of (1.3), (1.4) in $C^{4}([a, b])$.

(ii) $u \neq \theta \Phi(u)$ for all $\theta \in[0,1]$ and $u \in Z$ such that $\|u\|_{\infty}=r$ for sufficiently small $r>0$. Indeed, let $\alpha \in\left(0, c_{2}^{-1}\right)$, where $c_{2}$ is the constant in (3.1). By $\left(H_{4}\right)$ we can choose $r>0$ such that $f(s) \leqslant \alpha s$ for $0 \leq s \leq r$. Now suppose that there exist $\theta \in[0,1]$ and $u \in Z$ such that $u=\theta \Phi(u)$ with $\|u\|_{\infty}=r$. Then $\Delta^{2} u=\theta g f(u)$. With the notations of step 1 of the proof of Theorem 3.1, we have

$$
\begin{aligned}
\int_{a}^{b} t^{n-1} \varrho g u d t & =\int_{a}^{b} t^{n-1} u \Delta^{2} \varphi d t=\int_{a}^{b} t^{n-1} \varphi \Delta^{2} u d t \\
& =\theta \int_{a}^{b} t^{n-1} \varphi g f(u) d t \leq \alpha c_{2} \theta \int_{a}^{b} t^{n-1} \varrho g u d t
\end{aligned}
$$

and we reach a contradiction.

(iii) By $\left(H_{3}\right)$, there exists $\lambda>c_{1}^{-1}$ (where $c_{1}$ is the constant in (3.1)) and $x_{0}>0$ such that $f(t+x) \geq \lambda t$ for all $t \geq 0$ and $x \geq x_{0}$. Then using the same arguments as in step 1 of the proof of Theorem 3.1, we can show that the equation $F(u, x)=u$ has no solutions $u \in Z$ for $x \geq x_{0}$. 
(iv) Finally, using Remark 3.1 and (iii) above we can find a constant $R>r$ such that $F(u, x) \neq u$ for all $x \geq 0$ and $u \in Z$ with $\|u\|_{\infty}=R$.

Now we can apply Proposition 2.1 and Remark 2.1 stated in [7] to conclude that $\Phi$ has a nontrivial fixed point. The proof of the theorem is complete.

\section{PROOF OF THEOREM 1.2}

The proof of Theorem 1.2 is based on the maximum principle and the technique of moving parallel planes as in [9], [16] for second order equations and [5], [6] for fourth order equations. Subsequently $\Delta$ denotes equally the cartesian form and the polar form of the Laplacian. In the same way we write indifferently $u(x)$ or $u(|x|)$.

Assume that $\Delta u(a) \leq \Delta u(b)$ for some nonnegative nontrivial solution $u \in C^{4}([a, b])$ of problem (1.3), (1.4). Then by Theorem 2.1 we have $u>0$ in $\Omega(a, b)$ and $\Delta u<\Delta u(b)$ in $\Omega(a, b)$. Let $\lambda \in\left[\frac{a+b}{2}, b\right)$ and de-

fine $\Sigma(\lambda)=\Omega(a, b) \cap\left\{x=\left(x_{1}, x^{\prime}\right) \in \mathbb{R}^{n} ; x_{1}>\lambda\right\}$. Let $\Sigma^{\prime}(\lambda)$ denote the reflection of $\Sigma(\lambda)$ in the plane $T_{\lambda}=\left\{x=\left(x_{1}, x^{\prime}\right) \in \mathbb{R}^{n} ; x_{1}=\lambda\right\}$. Define the function

$$
u_{\lambda}(x)=u\left(2 \lambda-x_{1}, x^{\prime}\right) \quad \text { for } x \in \Sigma^{\prime}(\lambda) .
$$

We have the following Lemma.

Lemma 5.1. $u$ (resp. $\Delta u$ ) is strictly increasing (resp. strictly decreasing) as one enters $\Omega(a, b)$ from $\left\{x \in \mathbb{R}^{n} ;|x|=b\right\}$ along any nontangential direction $\vec{s}$, for some positive distance $d>0$ into $\Omega(a, b)$.

Proof. We have $u=\frac{\partial u}{\partial v}=0$ on $\partial \Omega(a, b)$. Since by Theorem $2.1 \Delta u$ is a positive constant on $\left\{x \in \mathbb{R}^{n} ;|x|=b\right\}$ the proof is immediate.

From Lemma 5.1 we deduce the existence of $\eta \in\left(0, \frac{a+b}{2}\right)$ such that, for $\lambda \in[b-\eta, b)$, we have 


$$
\left\{\begin{array}{l}
u_{\lambda}-u<0 \text { in } \Sigma^{\prime}(\lambda) \text { and } \frac{\partial u}{\partial x_{1}}<0 \text { in } \Sigma(\lambda) \\
\Delta\left(u_{\lambda}-u\right)>0 \text { in } \Sigma^{\prime}(\lambda) \text { and } \frac{\partial \Delta u}{\partial x_{1}}>0 \text { in } \Sigma(\lambda)
\end{array}\right.
$$

Decrease $\lambda$ until a critical value $\mu \geq(a+b) / 2$ is reached, beyond which (5.1) is no longer true. Then (5.1) holds for $\lambda \in(\mu, b)$ while for $\lambda=\mu$ we have by continuity

$$
\left\{\begin{array}{l}
u_{\mu}-u \leq 0 \text { in } \Sigma^{\prime}(\mu) \text { and } \frac{\partial u}{\partial x_{1}}<0 \text { in } \Sigma(\mu) \\
\Delta\left(u_{\mu}-u\right) \geq 0 \text { in } \Sigma^{\prime}(\mu) \text { and } \frac{\partial \Delta u}{\partial x_{1}}>0 \text { in } \Sigma(\mu) .
\end{array}\right.
$$

Suppose $\mu>(a+b) / 2$. We have $u_{\mu} \not \equiv$ in $\Sigma^{\prime}(\mu)$ since $u>0$ in $\Omega(a, b)$. The maximum principle ([10] p. 15) and the Hopf boundary lemma ([10] p. 33) imply that

$$
u_{\mu}-u<0 \text { in } \Sigma^{\prime}(\mu) \text { and } \frac{\partial u}{\partial x_{1}}<0 \text { on } T_{\mu} \cap \Omega(a, b)
$$

where the second inequality follows from the fact that $\frac{\partial}{\partial x_{1}}\left(u_{\mu}-u\right)=$ $-2 \frac{\partial u}{\partial x_{1}}$ on $T_{\mu} \cap \Omega(a, b)$. Now $\left(H_{5}\right)$ and $\left(H_{6}\right)$ imply that $\Delta^{2}\left(u_{\mu}-u\right) \leq 0$ in $\Sigma^{\prime}(\mu)$. From our assumption we have $\Delta u<\Delta u(b)$ in $\Omega(a, b)$. Thus $\Delta\left(u_{\mu}-u\right) \equiv 0$ in $\Sigma^{\prime}(\mu)$. The maximum principle and the Hopf boundary lemma imply that

$$
\Delta\left(u_{\mu}-u\right)>0 \text { in } \Sigma^{\prime}(\mu) \text { and } \frac{\partial \Delta u}{\partial x_{1}}>0 \text { on } T_{\mu} \cap \Omega(a, b)
$$

where the second inequality follows from the fact that $\frac{\partial}{\partial x_{1}}\left(\Delta\left(u_{\mu}-u\right)\right)=$ $-2 \frac{\partial \Delta u}{\partial x_{1}}$ on $T_{\mu} \cap \Omega(a, b)$. (5.2) and (5.3) show that (5.1) holds for $\lambda=\mu$.

Now our definition of $\mu$ implies that either there is a strictly increasing sequence $\left(\lambda_{j}\right)$ with $\lim _{j \rightarrow \infty} \lambda_{j}=\mu\left(\lambda_{j}>(a+b) / 2 \forall j\right)$ such that for each $j$ there is a point $x_{j} \in \Sigma^{\prime}\left(\lambda_{j}\right)$ for which 


$$
u_{\lambda_{j}}\left(x_{j}\right)-u\left(x_{j}\right) \geq 0 \quad \forall j
$$

or that there is a strictly increasing sequence $\left(\mu_{j}\right)$ with $\lim _{j \rightarrow \infty} \mu_{j}=\mu$ $\left(\mu_{j}>(a+b) / 2 \forall j\right)$ such that for each $j$ there is a point $z_{j} \in \Sigma^{\prime}\left(\mu_{j}\right)$ for which

$$
\Delta u_{u_{j}}\left(z_{j}\right)-\Delta u\left(z_{j}\right) \leq 0 \quad \forall j .
$$

In the situation (5.4), a subsequence which we still call $x_{j}$ will converge to some point $x \in \overline{\Sigma^{\prime}(\mu)}$; then $u_{\mu}(x)-u(x) \geq 0$. Since (5.1) holds for $\lambda=\mu$ we must have $x \in \partial \Sigma^{\prime}(\mu)$; If $x \in \partial \Sigma^{\prime}(\mu) \backslash T_{\mu}$ then $0=u_{\mu}(x)<u(x)$, a contradiction. Therefore $x \in T_{\mu}$. Using Lemma 5.1, (5.2) and (5.1) with $\lambda=\mu$ we see that for some $\varepsilon>0$ we have

$$
\frac{\partial u}{\partial x_{1}}<0 \text { in } \Omega(a, b) \cap\left\{x=\left(x_{1}, x^{\prime}\right) \in \mathbb{R}^{n} ; x_{1}>\mu-\varepsilon\right\} .
$$

The straight segment joining $x_{j}$ to its symmetric with respect to $T_{\lambda_{j}}$ belongs to $\Omega(a, b)$ and by the theorem of the mean it contains a point $y_{j}$ such that

$$
\frac{\partial u}{\partial x_{1}}\left(y_{j}\right) \geq 0
$$

Since $\lim _{j \rightarrow \infty} y_{j}=x$, we obtain a contradiction to (5.6).

In the situation (5.5), a subsequence which we still call $z_{j}$ will converge to some point $z \in \overline{\Sigma^{\prime}(\mu)}$; then $\Delta u_{\mu}(z)-\Delta u(z) \leq 0$. Since (5.1) holds for $\lambda=\mu$ we must have $z \in \partial \Sigma^{\prime}(\mu)$; If $z \in \partial \Sigma^{\prime}(\mu) \backslash T_{\mu}$ then $\Delta u(b)=\Delta u_{\mu}(z)>\Delta u(z)$, a contradiction. Therefore $z \in T_{\mu}$. Using Lemma $5.1,(5.3)$ and (5.1) with $\lambda=\mu$ we see that for some $\varepsilon>0$ we have

$$
\frac{\partial \underline{\Delta} \underline{u}}{\partial x_{1}}>0 \text { in } \Omega(a, b) \cap\left\{x=\left(x_{1}, x^{\prime}\right) \in \mathbb{R}^{n} ; x_{1}>\mu-\varepsilon\right\} .
$$

The straight segment joining $z_{j}$ to its symmetric with respect to $T_{\mu_{j}}$ belongs to $\Omega(a, b)$ and by the theorem of the mean it contains a point $t_{j}$ such that

$$
\frac{\partial \underline{\Delta} \underline{u}}{\partial x_{1}}\left(t_{j}\right) \leq 0
$$

Since $\lim _{j \rightarrow \infty} t_{j}=z$, we obtain a contradiction to $(5,7)$. 
Thus we have proved that $\mu=(a+b) / 2$ and that (5.1) holds for $\lambda \epsilon$ $\left(\frac{a+b}{2}, b\right)$. By continuity we have

$$
u_{\varrho}-u \leq 0 \text { in } \Sigma^{\prime}(\varrho) \text { and } \frac{\partial u}{\partial x_{1}}<0 \text { in } \Sigma(\varrho)
$$

and

$$
\Delta\left(u_{e}-u\right) \geq 0 \text { in } \Sigma^{\prime}(\varrho) \text { and } \frac{\partial \Delta u}{\partial x_{1}}>0 \text { in } \Sigma(\varrho)
$$

where $Q=\frac{a+b}{2}$. Now let $x=(a, 0)$ then

$$
\left(u_{e}-u\right)(x)=\frac{\partial}{\partial v}\left(u_{e}-u\right)(x)=0
$$

and the Hopf lemma implies that $u_{e}-u \equiv 0$ in $\Sigma^{\prime}(\varrho)$, but this is impossible. The proof of the theorem is complete.

\section{References}

[1] D. ARCOYA: Positive solutions for semilinear Dirichlet problems in an annulus, J. Differential Equations 94 (1991), 217-227.

[2] C. BANDLE and M. KWONG: Semilinear elliptic problems in annular domains, J. Appl. Math. Phys. 40 (1989), 245-257.

[3] C. Bandle, C. V. CofFMAN and M. MARCus: Nonlinear elliptic problems in annular domains, J. Differential Equations 69 (1987), 322-345.

[4] C. V. CoffMAN and M. Marcus: Existence and uniqueness results for semilinear Dirichlet problems in annuli, Arch. Ration. Mech. Analysis 108 (1989), 293-307.

[5] R. DALMASSo: Symmetry properties of solutions of some fourth order ordinary differential equations, Bull. Sc. Math., $2^{*}$ série, 117 (1993), 441-462.

[6] R. DALmasso: Un problème de symétrie pour une équation biharmonique, Annales de la Faculté des Sciences de Toulouse, vol. XI, n. ${ }^{\circ} 3,(1990) 45-$ 53.

[7] D. De Figueiredo, P. L. Lions and R. D. Nussbaum: A priori estimates and existence of positive solutions of semilinear elliptic equations, J. Math. Pures Appl. 61 (1982), 41-63.

[8] X. GARAIZAR: Existence of positive radial solutions for semilinear elliptic equations in the annulus, J. Differential Equations 70 (1987), 69-92.

[9] B. GidAs, W.-M. Ni and L. NIRENBERG: Symmetry and related properties via the maximum principle, Comm. Math. Phys., 68 (1979), 209-243. 
[10] D. GILBARG and N. S. TRUDINGER: Elliptic partial differential equations of second order, Springer-Verlag, Vol. 224, Berlin-Heidelberg-New York, 1977.

[11] S. S. LIN: On the existence of positive radial solution for nonlinear elliptic equations in annular domains, J. Differential Equations 81 (1989), 221-233.

[12] S. S. LIN and F. M. PAI: Existence and multiplicity of positive radial solutions for semilinear elliptic equations in annular domains, SIAM J. Math. Anal., Vol. 22, n. ${ }^{\circ} 6$ (1991), 1500-1515.

[13] M. A. NAIMARK: Elementary theory of linear differential operators Part I, F. UNGAN, New York, 1967.

[14] M. PROTTER and H. WeINBERGER: Maximum principles in differential equations, Prentice Hall, 1967.

[15] J. SaNTANILla: Existence and nonexistence of positive radial solutions for some semilinear elliptic problems in annular domains, Nonlinear Anal. 16 (1991) 861-879.

[16] J. SERRIN: A symmetry problem in potential theory, Arch. Ration. Mech. Analysis 43 (1971), 304-318.

\author{
Laboratoire LMC-IMAG \\ Equipe EDP \\ Tour IRMA-BP 5 \\ F. 38041 Grenoble Cedex 9 \\ France \\ E-mail: Dalmasso @ imag. fr
}

